\title{
铜催化苯甲酰亚胺高烯丙酯的分子内胺化全氟烷基化反应
}

\author{
张衡牟学清陈弓* 何刚* \\ (南开大学化学学院 元素有机化学国家重点实验室 天津 300071)
}

\begin{abstract}
摘要 报道了铜催化苯甲酰亚胺高烯丙酯底物的分子内胺化全氟烷基化反应. 该反应以全氟碘代烷为全氟烷基化试 剂, 醋酸铜为催化剂, 邻菲啰啉为配体, 在醋酸银存在下以中等的收率实现苯甲酰亚胺高烯丙酯底物末端双键的胺化 全氟烷基化, 最终生成 1,3-噁嗪类分子. 多种官能团取代的苯甲酰亚胺高烯丙酯和具有不同碳链长度的全氟碘代烷烃 都能适用于该反应，为多氟烷基取代的 1,3-噁嗪类化合物的合成提供了一种简洁的方法. 多氟烷基取代的 1,3-噁嗪类化 合物还可在温和条件下高效转化为 $\gamma$ 氨基醇衍生物. 初步的机理研究证明该反应经历了全氟烷基自由基对碳碳双键的 亲电加成, 之后苯甲酰亚胺基团作为分子内亲核性胺源经历分子内亲核取代途径生成 1,3-啞嗪骨架.
\end{abstract}

关键词 苯甲酰亚胺高烯丙酯; 全氟碘代烷烃; 胺化全氟烷基化反应; 1,3-啞嗪; 铜催化

\section{Copper-catalyzed Intramolecular Aminoperfluoroalkylation Reaction of O-Homoallyl Benzimidates}

\author{
Zhang, Heng Mou, Xueqing Chen, Gong* He, Gang* \\ (State Key Laboratory and Institute of Elemento-Organic Chemistry, College of Chemistry, Nankai University, Tianjin \\ 300071)
}

\begin{abstract}
Azaheterocycles have been broadly applied in the development of therapeutic agents, agrochemicals and functional material molecules. Azaheterocycles equipped with perfluoroalkyl group usually manifest superior physical and biological properties than their parent molecules, such as showing improved metabolic stability and high lipophilicity. The synthesis of perfluoroalkyl modified azaheterocycles has attracted considerable research interest in recent years. The strategy of intramolecular aminoperfluoroalkylation of alkenes, which functionalize $\mathrm{C}=\mathrm{C}$ bond with an external perfluoroalkyl group and an internal amine nucleophile in one pot, provides a streamlined synthesis of perfluoroalkyl substituted azaheterocycles. This strategy has been applied by Liu, Sodeoka and other research groups in the synthesis of perfluoroalkyl substituted aziridines, pyrrolidines, lactams and pyrazolines featuring the use of pendent amine, amide, hydrazone or urea group as internal amine source. We have previously developed a copper(I)-catalyzed intramolecular aminotrifluoromethylation reaction of $O$-homoallyl benzimidates with Togni reagent I for the synthesis of trifluoromethyl containing chiral 1,3-oxazines using a chiral BOX ligand. However, this method is limited to aminotrifluoromethylation reaction as other perfluoroalkyl substituted hypervalent iodine reagents are not easily accessible. Herein, we report our recent research results on the synthesis of perfluoroalkyl substituted 1,3-oxazines using commercial available perfluoroalkyl iodides as perfluoroalkyl source. This intramolecular aminoperfluoroalkylation reaction proceeds selectively in the presence of $\mathrm{Cu}(\mathrm{OAc})_{2}$ catalyst, 1,10-phenanthroline ligand and $\mathrm{AgOAc}$ additive. A broad range of $O$-homoallyl benzimidates and perfluoroalkyl iodides are compatible with the reaction conditions, affording perfluoroalkyl substituted 1,3-oxazines in moderate to good yields. The 1,3-oxazine product can be prepared in gram scale and readily hydrolyzed under mild conditions to give perfluoroalkyl substituted 1,3-amino alcohols. Preliminary mechanism studies revealed that this intramolecular aminoperfluoroalkylation reaction initiated with the addition of a perfluoroalkyl radical to the terminal alkene, and the subsequent functionalization with the benzimidate motif via intramolecular substitution generated 1,3-oxazine products.

Keywords $O$-homoallyl benzimidates; aminoperfluoroalkylation; perfluoroalkyl iodides; 1,3-oxazines; copper catalysis
\end{abstract}

\section{1 引言}

氟原子具有较小的原子半径和较大的电负性，其与
碳原子形成的碳氟键具有较强的键能和化学稳定性 ${ }^{[1]}$. 这些特殊的理化性质使得含氟化合物在材料和医药开 发领域得到了广泛的应用. 例如, 引入氟原子或多氟烷

\footnotetext{
*E-mail: gongchen@nankai.edu.cn; hegang@nankai.edu.cn

Received June 18, 2019; published August 13, 2019.

Supporting information for this article is available free of charge via the Internet at http://sioc-journal.cn.

Project supported by the National Natural Science Foundation of China (21672105, 21702109, 21890722), Natural Science Foundation of Tianjin (17JCYBJC19700, 18JCZDJC32800), and the Fundamental Research Funds for the Central Universities (Nankai University (No. 63161122)). We dedicate this work to the $100^{\text {th }}$ anniversary of Nankai University.

项目受国家自然科学基金(Nos. 21672105, 21702109, 21890722)、天津市自然科学基金(Nos. 17JCYBJC19700, 18JCZDJC32800)和南开大学中央高校基 本科研业务费专项资金(No. 63161122)资助. 谨以此文恭贺南开大学百年华诞.
} 
基能改变母体化合物的偶极矩、亲脂性和化学稳定性等 理化性质，是改善母体化合物生物活性的重要策略 ${ }^{[2]}$. 氮杂环骨架是众多医药分子的核心结构, 因此多氟烷基 取代的氮杂环骨架的合成近年来受到了广泛的关注 ${ }^{[3]}$. 通过碳碳双键的分子内胺化多氟烷基化反应能在引入 多氟烷基官能团的同时构筑氮杂环骨架，是制备多氟烷 基取代氮杂环化合物的高效策略之一 ${ }^{[4,5]}$. Sodeoka ${ }^{[6 a \sim 6 c]}$ 和刘心元 ${ }^{[6 \mathrm{~d} \sim 6 \mathrm{f}, 7]}$ 等课题组在这一领域做出了开创性的工 作，通过在烯烃类分子中预先引入氨基、嫝等含氮亲核 片段, 发展了铜催化的分子内胺化多氟烷基化反应, 实 现了多种全氟烷基取代的氮杂环化合物的合成(图 1A). 我们课题组在之前工作中发现酰亚胺酯片段可以作为 分子内亲核性胺源 ${ }^{[8]}$, 发展了脂肪醇骨架的分子内碳氢 键氨基化反应 $^{[9,10]}$. 受此启发, 我们采用 Togni 试剂作为 三氟甲基源, 在铜催化剂和手性双齿噁唑啉配体调控 下, 实现了酰亚胺高烯丙酯的对映选择性分子内胺化三 氟甲基化反应，合成了三氟甲基取代的 1,3-噁嗪类化合 物(图 1B $)^{[11]}$. Togni 试剂的制备繁琐, 且局限于三氟甲基 化转化 ${ }^{[12]}$. 因此, 发展更廉价易得、种类丰富的氟烷基 化试剂参与的胺化全氟烷基化反应仍然值得进一步探 索. 全氟碘代烷烃是一类在化工领域广泛使用的大宗化

(A) Copper catalyzed intramolecular aminoperfluoroalkylation of unactivated alkene

$$
\begin{aligned}
& \text { perfluoroalkyl ( } \mathrm{R}_{\mathrm{f}} \text { ) source } \\
& \text { arylamine amide hydrazone }
\end{aligned}
$$

(B) Aminotrifluoromethylation of O-homoallyl benzimidates with Togni reagent I
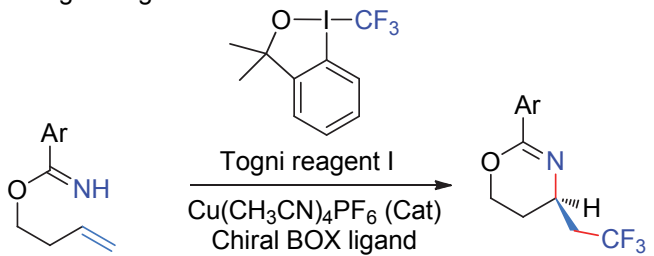

O-homoallyl benzimidate

up to $94 \%$ ee up to $88 \%$ yield

(C) Aminoperfluoroalkylation of O-homoallyl benzimidates with perfluoroalkyl iodides

$$
\mathrm{Cu}(\mathrm{OAc})_{2}(10 \mathrm{~mol} \%)
$$

1,10-phenanthroline (10 mol\%)
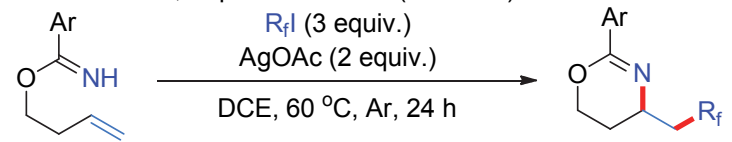

O-homoallyl benzimidate

1,3-oxazine

图 1 分子内胺化多氟烷基化反应制备多氟烷基取代的氮杂环骨架

Figure 1 Perfluoroalkylative intramolecular difunctionalization of unactivated alkene
学品, 品种多样且商业可得 ${ }^{[13]}$. 陈庆云等 ${ }^{[14]}$ 课题组系统 研究了全氟碘代烷烃在过渡金属催化或者光照等条件 下产生全氟烷基自由基的历程，并与烯烃、富电子芳环 等亲电物种发生加成反应实现了全氟烷基的引入 ${ }^{[15 \sim 17]}$. 在此基础上，我们发展了全氟碘代烷参与的苯甲酰亚胺 高烯丙酯衍生物的分子内胺化全氟烷基化反应，为全氟 烷基取代的 1,3-噁嗪类化合物的制备提供了新的方法 (图 1C) ${ }^{[18] .}$

\section{2 结果与讨论}

\section{1 反应条件优化}

首先采用苯甲酰亚胺高烯丙酯 $\mathbf{1}$ 为模板底物, 全氟 碘代丁烷为氟烷基化试剂对反应条件进行了考察(表 1). 在之前的三氟甲基化条件下(Entry 1)预期反应不能发 生, 大部分原料剩余. 经过一系列的尝试, 发现加入 2

表 1 反应条件优化

Table 1 Reaction condition optimization

Cat. (10 mol\%)

\begin{tabular}{|c|c|c|c|c|}
\hline Entry & Catalyst & Additive & Solvent & Yield $^{a} / \%$ of 2 \\
\hline $1^{b}$ & $\mathrm{Cu}\left(\mathrm{CH}_{3} \mathrm{CN}\right)_{4} \mathrm{PF}_{6}$ & BOX ligand & DCE & NR \\
\hline 2 & $\mathrm{Cu}\left(\mathrm{CH}_{3} \mathrm{CN}\right)_{4} \mathrm{PF}_{6}$ & $\mathrm{AgOAc}$ & DCE & 44 \\
\hline 3 & $\mathrm{CuCl}$ & $\mathrm{AgOAc}$ & DCE & 54 \\
\hline 4 & $\mathrm{CuBr}$ & $\mathrm{AgOAc}$ & DCE & 62 \\
\hline 5 & $\mathrm{CuI}$ & $\mathrm{AgOAc}$ & DCE & 60 \\
\hline 6 & $\mathrm{Cu}(\mathrm{acac})_{2}$ & $\mathrm{AgOAc}$ & DCE & 54 \\
\hline 7 & $\mathrm{Cu}(\mathrm{OTf})_{2}$ & $\mathrm{AgOAc}$ & DCE & 45 \\
\hline 8 & $\mathrm{Cu}(\mathrm{OAc})_{2}$ & $\mathrm{AgOAc}$ & DCE & $72(64)^{c}$ \\
\hline 9 & $\mathrm{Cu}(\mathrm{OAc})_{2}$ & AgOTFA & DCE & 19 \\
\hline 10 & $\mathrm{Cu}(\mathrm{OAc})_{2}$ & AgOTf & DCE & 47 \\
\hline 11 & $\mathrm{Cu}(\mathrm{OAc})_{2}$ & $\mathrm{Ag}_{2} \mathrm{CO}_{3}$ & DCE & 53 \\
\hline 12 & $\mathrm{Cu}(\mathrm{OAc})_{2}$ & $\mathrm{Cs}_{2} \mathrm{CO}_{3}$ & DCE & 20 \\
\hline 13 & $\mathrm{Cu}(\mathrm{OAc})_{2}$ & $\mathrm{AgOAc}$ & $\mathrm{CH}_{3} \mathrm{CN}$ & 67 \\
\hline 14 & $\mathrm{Cu}(\mathrm{OAc})_{2}$ & $\mathrm{AgOAc}$ & THF & 52 \\
\hline 15 & $\mathrm{Cu}(\mathrm{OAc})_{2}$ & $\mathrm{AgOAc}$ & EtOAc & 63 \\
\hline 16 & $\mathrm{Cu}(\mathrm{OAc})_{2}$ & - & DCE & NR \\
\hline 17 & & $\mathrm{AgOAc}$ & DCE & $<5$ \\
\hline $18^{d}$ & $\mathrm{Cu}(\mathrm{OAc})_{2}$ & $\mathrm{AgOAc}$ & DCE & $<5$ \\
\hline $19^{e}$ & $\mathrm{Cu}(\mathrm{OAc})_{2}$ & $\mathrm{AgOAc}$ & DCE & 53 \\
\hline
\end{tabular}

1,10-phenanthroline (10 mol\%)

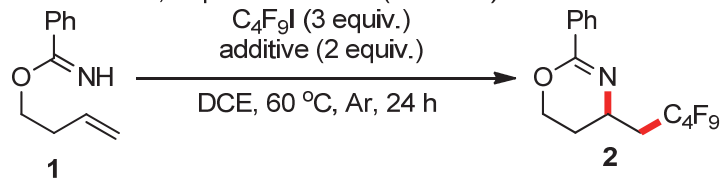

${ }^{a}$ Yields are based on ${ }^{1} \mathrm{H}$ NMR analysis of reaction mixture with $1,1,2,2-$ tetrachloroethane as internal standard on a $0.2 \mathrm{mmol} \mathrm{scale}{ }^{b}$ See Supporting Information for detailed results. ${ }^{c}$ Isolated yield on a $0.2 \mathrm{mmol}$ scale. ${ }^{d}$ The reaction was conducted in air. ${ }^{e}$ Without 1,10 -phenanthroline. NR: no reaction, and most of 1 was recovered. 
equiv. 的 $\mathrm{AgOAc}$ 能有效地促进反应的进行, 以中等的收 率得到产物 2 (Entry 2). 之后通过对反应的其他因素进 行考察, 我们发现: (1)一价铜和二价铜试剂都能催化反 应的进行, 其中 $\mathrm{Cu}(\mathrm{OAc})_{2}$ 给出最优的转化率, 能以 $72 \%$ 的核磁收率和 $64 \%$ 的分离收率得到目标产物 2 (Entries 2 8). (2) AgOAc 是最优的添加剂, 采用其他银盐或者 碳酸铯时, 反应收率大幅下降(Entries 9～12). (3)反应 在乙腈、四氢呋喃等其他溶剂中给出稍低的收率(Entries 13 15). (4)反应在空气气氛下只能产生少量产物, 大部 分原料末能转化(Entry 18).

\section{2 底物拓展}

在得到最优的反应条件后(Table 1, Entry 8), 使用 全氟碘代丁烷为氟烷基化试剂对苯甲酰亚胺高烯丙酯 类底物的适用范围进行了考察(图 2). 苯环上带有给电 子取代基(如甲基、甲氧基，3 6) 和缺电子取代基(如硝 基，10)的底物都适用于该反应，能以中等的收率得到相 应的 1,3-噁嗪类化合物. 反应收率不高的原因主要是因 为部分原料剩余以及少量原料发生水解反应. 苯环上带 有卤素(氯、溴、碘, 7 9)的底物在标准条件下也能以

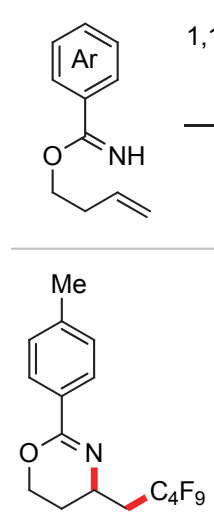

$\mathrm{Cu}(\mathrm{OAc})_{2}(10 \mathrm{~mol} \%)$

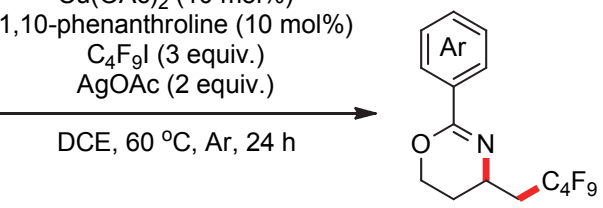

3, $50 \%$

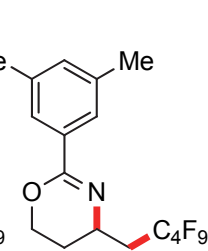

5, $68 \%$

\section{6, $63 \%$}<smiles>Cc1cccc(C2=N[C@H](CC(F)(F)F)CCO2)c1</smiles>

4, $69 \%$

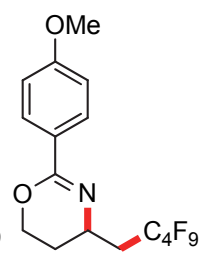<smiles>FC(F)(F)CC1CCOC(c2ccc(Cl)cc2)=N1</smiles>

7, $72 \%$<smiles>FC(F)(F)CC1CCOC(c2ccc(Br)cc2)=N1</smiles>

8, $68 \%$

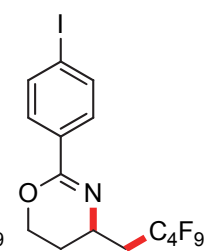

9, 65\%<smiles>FC(F)(F)CC1CCOC(c2ccc3ccccc3c2)=N1</smiles>

$11,69 \%$

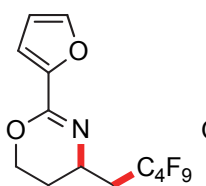

$12,32 \%$

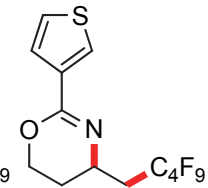

$13,52 \%$

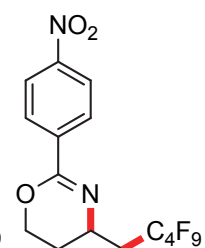

$10,47 \%$

图 2 苯甲酰亚胺高烯丙酯类底物的适用范围

Figure 2 Scope of $O$-homoallyl benzimidate substrates All yields are based on isolated product on a $0.2 \mathrm{mmol}$ scale

中等偏上的收率给出相应产物, 为分子结构的进一步修 饰和转化提供了便利. 䒬环(11)或者呋喃(12)、噻吩 $(13)$ 等杂芳环取代的底物也适用于该反应，能以中等的收率 得到相应的产物. 然而, 带有多取代双键的苯甲酰亚胺 高烯丙酯类底物和三氯乙酰亚胺高烯丙酯(14)底物，在 最优反应条件下未能得到相应成环产物 ${ }^{[19]}$. 如图 3 所 示，具有不同碳链长度的全氟碘代烷烃如 $\mathrm{C}_{3} \mathrm{~F}_{7} \mathrm{I}, \mathrm{C}_{5} \mathrm{~F}_{11} \mathrm{I}$, $\mathrm{C}_{6} \mathrm{~F}_{13} \mathrm{I}$ 和 $\mathrm{C}_{8} \mathrm{~F}_{17} \mathrm{I}$ 都适用于该铜催化的分子内氨化全氟烷 基化反应，以中等收率得到全氟烷基取代的 1,3-噁嗪类 化合物.

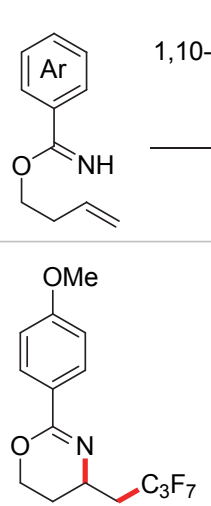

$15,56 \%$

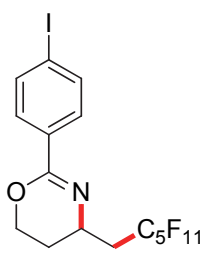

$18,70 \%$<smiles>Cc1cccc(C2=NC(CC(F)F)CCO2)c1</smiles>

$21,56 \%$
$\mathrm{Cu}(\mathrm{OAc})_{2}(10 \mathrm{~mol} \%)$

10-phenanthroline (10 $\mathrm{mol} \%)$

$\mathrm{C}_{n} \mathrm{~F}_{2 n+1} \mathrm{I}$ (3 equiv.) AgOAc (2 equiv.)

DCE, $60^{\circ} \mathrm{C}, \mathrm{Ar}, 24 \mathrm{~h}$

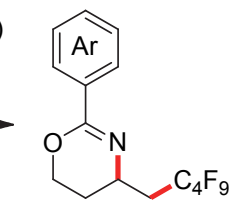

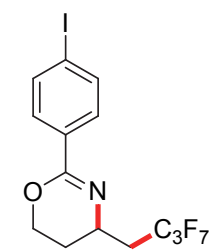

$16,68 \%$

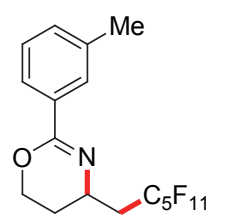

$17,60 \%$

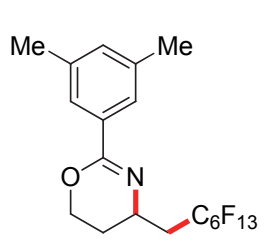

$19,62 \%$

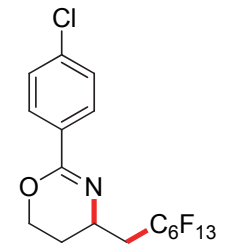

$20,57 \%$

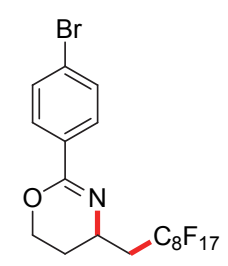

22, $61 \%$
图 3 全氟碘代烷烃的适用范围

Figure 3 Scope of perfluoroalkyl iodides

All yields are based on isolated product on a $0.2 \mathrm{mmol}$ scale

\section{3 反应实用性考察}

为了考察该铜催化分子内氨化全氟烷基化反应的 实用性，首先尝试了反应产物的克级制备(图 4). 当用 $0.8 \mathrm{~g}$ 原料 23 反应时，以 $64 \%$ 的收率得到多氟烷基取代 的 1,3-噁嗪产物 $4(1.1 \mathrm{~g})$. 1,3-噁嗪产物在温和条件下可 以发生开环反应, 生成在有机合成中应用广泛的 $\gamma$-氨基 醇衍生物。

\section{4 反应机理研究}

\subsection{1 反应机理假设}

根据已有文献和观察到的具体实验现象 ${ }^{[6]}$, 提出了 


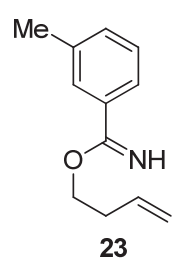

$0.8 \mathrm{~g}, 4.23 \mathrm{mmol}$
$\mathrm{Cu}(\mathrm{OAc})_{2}(10 \mathrm{~mol} \%)$

1,10-phenanthroline (10 mol\%)

$\mathrm{C}_{4} \mathrm{~F}_{9} \mathrm{l}$ (3 equiv.)

$\mathrm{AgOAc}$ (2 equiv.)

DCE, $60^{\circ} \mathrm{C}, \mathrm{Ar}, 24 \mathrm{~h}$

\section{(1)}

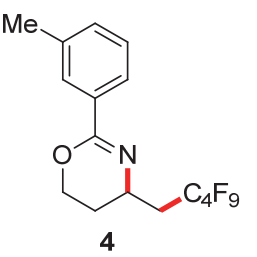

$1.1 \mathrm{~g}, 64 \%$<smiles>FC(F)(F)CC1CCOC(c2ccccc2)=N1</smiles>

2
(1) aq. $\mathrm{HCl}$ ( $1 \mathrm{~mol} / \mathrm{L}, 10$ equiv.)

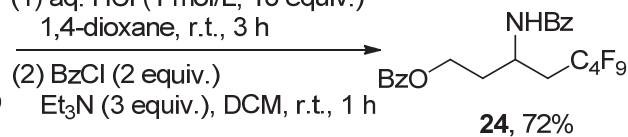

24, $72 \%$
图 4 克级反应和产物的进一步转化

Figure 4 Gram scale reaction and transformation of 1,3-oxazine

如图 5A 所示的反应机理. 二价铜首先发生歧化反应生 成少量一价铜和三价铜. 全氟碘代烷与一价铜发生单电 子转移反应, 之后碳碘键均裂产生全氟烷基自由基中间 体 $\mathrm{R}_{\mathrm{f}}$. 该自由基中间体与底物 1 中的末端双键发生区 域选择性的自由基加成反应，生成碳自由基中间体 $\mathbf{I}$. 该中间体可能经过下面三种途径最终生成目标产物: (a) 碳自由基中间体 I 与全氟碘代烷反应, 生成碘代中间体 II 和全氟烷基自由基, 碘代中间体 II 经过分子内亲核取 代反应得到成环产物; (b)碳自由基中间体 I 可被二价铜 氧化生成碳正离子中间体 III, 经过后续的分子内成环 反应得到产物; (c)中间体 I 还可以被反应体系中的二价 铜捕获, 形成三价铜中间体 IV, 最后经过还原消除反应 得到产物.

\subsection{2 机理实验}

为了验证反应的历程, 进行了初步的机理验证试验 (图 5B). 在标准反应条件下向反应体系中加入自由基抑 制剂四甲基哌啶氮氧化物(TEMPO)和 2,6-二叔丁基-4甲基苯酚(BHT)时, 反应被严重抑制，只能得到微量的 产物. 通过氟谱分析观测到了九氟丁基自由基被 TEMPO 捕获的产物 25, 从而证实了该反应的启动经历 自由基反应历程. 当采用烯丙基苯 26 作反应物时，以中 等收率得到了双键碘代全氟烷基化的产物 27. 通过其 他途径制备了碘代中间体 28, 化合物 28 在标准反应条 件下可顺利发生分子内亲核取代反应得到成环产物 2. 这一结果表明铜催化苯甲酰亚胺高烯丙酯的氨化全氟 烷基化反应很可能经历了反应路径 $\mathrm{a}$, 首先生成中间体 II, 之后在银盐的作用下发生分子内关环反应得到目标 产物.

\section{3 结论}

使用廉价易得的全氟碘代烷烃为全氟烷基化试剂, 发展了铜催化的苯甲酰亚胺高烯丙酯的分子内胺化全 氟烷基化反应. 该反应有较好的底物适用范围和官能团 兼容性, 克服了之前使用 Togni 试剂难以多样化的缺陷,

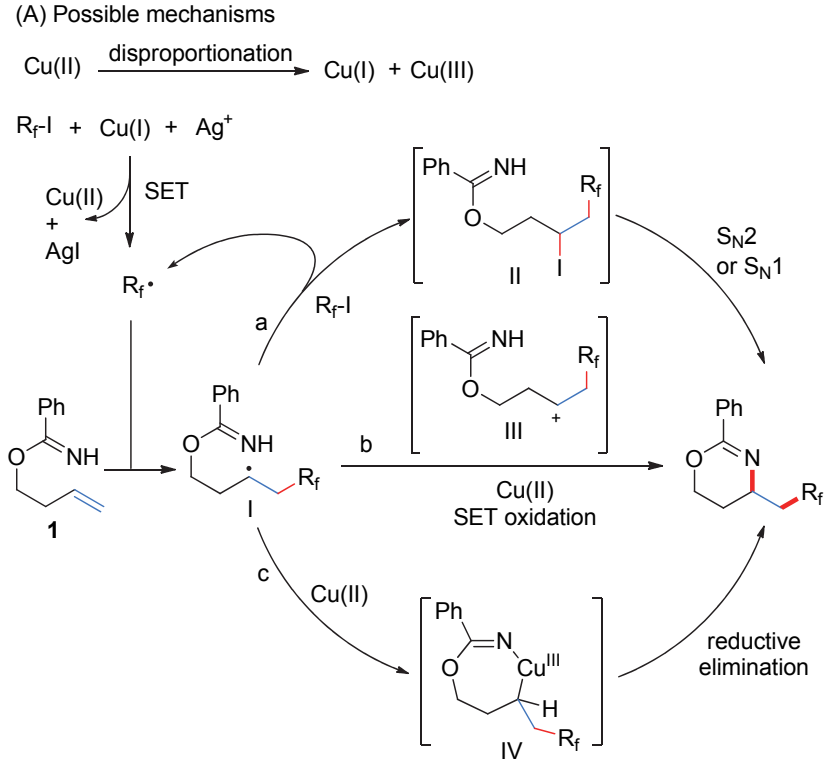

(B) Mechanistic experiments

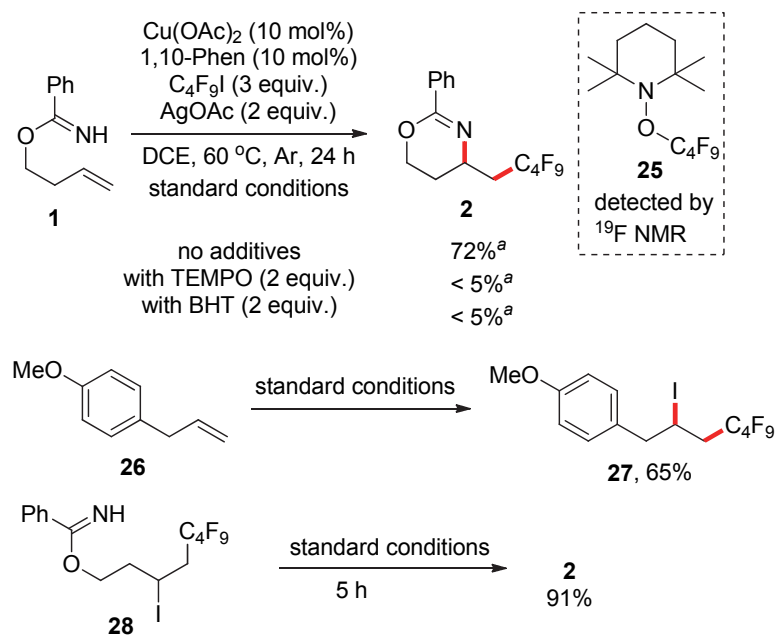

图 5 反应可能机理

Figure 5 Proposed mechanism

${ }^{a}$ Yields are based on ${ }^{1} \mathrm{H}$ NMR analysis of reaction mixture with 1,1,2,2tetrachloroethane as internal standard on a $0.2 \mathrm{mmol}$ scale

为合成多氟烷基取代的 1,3-噁嗪类化合物提供了实用的 新方法.

\section{References}

[1] Hu, J.; Ding, K. Acta Chim. Sinica 2018, 76, 905 (in Chinese). (胡金 波, 丁奎岭, 化学学报, 2018, 76, 905.)

[2] (a) Smart, B. E. J. Flurorine Chem. 2001, 109, 3; (b) Hagmann, W. K. J. Med. Chem. 2008, 51, 4359; (c) Purser, S.; Moore, P. R.; Swallow, S.; Gouverneur, V. Chem. Soc. Rev. 2008, 37, 320; (d) Zhou, Y.; Wang, J.; Gu, Z.; Wang, S.; Zhu, W.; Acena, J. L.; Soloshonok, V. A.; Izawa, K.; Liu, H. Chem. Rev. 2016, 116, 422.

[3] (a) Vitaku, E.; Smith, D. T.; Njardarson, J. T. J. Med. Chem. 2014, 57, 10257; (b) Meyer, F. Chem. Commun. 2016, 52, 3077.

[4] Tian, Y.; Chen, S.; Gu, Q.-S.; Lin, J.-S.; Liu, X.-Y. Tetrahedron Lett. 2018, 59, 203.

[5] (a) Takamasa, F.; Yoshiko, S.; Hisao, U. Chem. Lett. 1987, 16, 521; (b) Kim, E.; Choi, S.; Kim, H.; Cho, E. J. Chem.-Eur. J. 2013, 19, 6209; (c) Matcha, K.; Antonchick, A. P. Angew. Chem., Int. Ed. 2014, 53, 11960; (d) Wei, Q.; Chen, J.-R.; Hu, X.-Q.; Yang, X.-C.; 
Lu, B.; Xiao, W.-J. Org. Lett. 2015, 17, 4464; (e) Jarrige, L.; Carboni, A.; Dagousset, G.; Levitre, G.; Magnier, E.; Masson, G. Org. Lett. 2016, 18, 2906.

[6] For copper catalyzed intramolecular aminoperfuoroalkylation, see: (a) Egami, H.; Kawamura, S.; Miyazaki, A.; Sodeoka, M. Angew. Chem., Int. Ed. 2013, 52, 7841; (b) Kawamura, S.; Egami, H.; Sodeoka, M. J. Am. Chem. Soc. 2015, 137, 4865; (c) Kawamura, S.; Dosei, K.; Valverde, E.; Ushida, K.; Sodeoka, M. J. Org. Chem. 2017, 82, 12539; (d) Lin, J.-S.; Liu, X.-G.; Zhu, X.-L.; Tan, B.; Liu, X.-Y. J. Org. Chem. 2014, 79, 7084; (e) Lin, J.-S.; Xiong, Y.-P.; Ma, C.-L.; Zhao, L.-J.; Tan, B.; Liu, X.-Y. Chem.-Eur. J. 2014, 20, 1332; (f) Li, X.-F.; Lin, J.-S.; Liu, X.-Y. Synthesis 2017, 49, 4213; (g) Shen, K.; Wang, Q. Org. Chem. Front. 2016, 3, 222; (h) Yu, L.-Z.; Wei, Y.; Shi, M. Chem. Commun. 2016, 52, 13163; (i) Zhang, H.-Y.; Huo, W.; Ge, C.; Zhao, J.; Zhang, Y. Synlett 2017, 28, 962; (j) Chang, B.; Su, Y.; Huang, D.; Wang, K.-H.; Zhang, W.; Shi, Y.; Zhang, X.; Hu, Y. J. Org. Chem. 2018, 83, 4365.

[7] For enantioselective aminotrifluoromethylation of alkene, see: (a) Lin, J.-S.; Dong, X.-Y.; Li, T.-T.; Jiang, N.-C.; Tan, B.; Liu, X.-Y. J. Am. Chem. Soc. 2016, 138, 9357; (b) Lin, J.-S.; Wang, F.-L.; Dong, X.-Y.; He, W.-W.; Yuan, Y.; Chen, S.; Liu, X.-Y. Nat. Commun. 2017, 8, 14841.

[8] For recent examples of using imidates as nucleophile, see: (a) Brindle, C. S.; Yeung, C. S.; Jacobsen, E. N. Chem. Sci. 2013, 4, 2100; (b) Zhu, R.; Yu, K.; Gu, Z. Org. Biomol. Chem. 2014, 12, 6653.

[9] Mou, X.-Q.; Chen, X.-Y.; Chen, G.; He, G. Chem. Commun. 2018, $54,515$.

[10] For selected examples of intramolecular $\mathrm{C}-\mathrm{H}$ amination of imidates by other groups, see: (a) Wappes, E. A.; Nakafuku, K. M.; Nagib, D. A. J. Am. Chem. Soc. 2017, 139, 10204; (b) Stateman, L. M.; Wappes, E. A.; Nakafuku, K. M.; Edwards, K. M.; Nagib, D. A. Chem. Sci. 2019, 10, 2693; (c) Shaw, M.; Kumar, A. Org. Lett. 2019, 21,3108 .

[11] Mou, X.-Q.; Rong, F.-M.; Zhang, H.; Chen, G.; He, G. Org. Lett. 2019, 21, 4657.

[12] (a) Eisenberger, P.; Gischig, S.; Togni, A. Chem.-Eur. J. 2006, 12, 2579; (b) Matoušek, V.; Pietrasiak, E.; Schwenk, R.; Togni, A. J. Org. Chem. 2013, 78, 6763; (c) Charpentier, J.; Früh, N.; Togni, A. Chem. Rev. 2015, $115,650$.

[13] For selected reviews on the synthesis and application of perfluoroalkyl iodides, see: (a) Huang, B. Chin. J. Org. Chem. 1981, 1, 403 (in Chinese). (黄炳南, 有机化学, 1981, 1, 403.); (b) Brace, N. O. J. Flurorine Chem. 1999, 93, 1; (c) Brace, N. O. J. Flurorine Chem. 1999, 96, 101; (d) Brace, N. O. J. Flurorine Chem. 2001, 108, 147; (e) Murphy, P. M.; Baldwin, C. S.; Buck, R. C. J. Flurorine Chem. 2012, 138, 3; (f) Huang, H.; Wang, X.; Wang, J. Chin. J. Org. Chem. 2019, 39, 1 (in Chinese). (黄航, 王兮, 王剑波, 有机化学, 2019, 39, 1.)

[14] (a) Chen, Q.-Y.; Chen, Y.-X.; Huang, W.-Y. Acta Chim. Sinica 1984, 42, 906 (in Chinese). (陈庆云, 陈亚雄, 黄维垣, 化学学报, 1984, 42, 906.); (b) Chen, Q.-Y.; Yang, Z.-Y. J. Flurorine Chem. 1985, 28, 399; (c) Chen, Q.-Y.; Yang, Z.-Y. Acta Chim. Sinica 1985, 43, 1073 (in Chinese). (陈庆云, 杨震宇, 化学学报, 1985, 43, 1073.); (d) Chen, Q.-Y.; Yang, Z.-Y. Acta Chim. Sinica 1985, 43, 1118 (in Chinese). (陈庆云, 杨震宇, 化学学报, 1985, 43, 1118.); (e) Chen, Q.-Y.; Yang, Z.-Y. Acta Chim. Sinica 1986, 44, 265 (in Chinese). (陈
庆云, 杨震宇, 化学学报, 1986, 44, 265.); (f) Chen, Q.-Y.; Yang, Z.-Y. Acta Chim. Sinica 1986, 44, 1025 (in Chinese). (陈庆云, 杨震 宇, 化学学报, 1986, 44, 1025.); (g) Chen, Q.-Y.; Qiu, Z.-M. Acto Chim. Sinica 1987, 45, 354 (in Chinese). (陈庆云, 装再明, 化学学 报, 1987, 45, 354.); (h) Chen, Q.-Y.; Qiu, Z.-M. Acta Chim. Sinica 1988, 46, 258 (in Chinese). (陈庆云, 表再明, 化学学报, 1988, 46, 258.); (i) Chen, Q.-Y.; Chen, J.-G. Acta Chim. Sinica 1988, 46, 301 (in Chinese). (陈庆云, 杨建国, 化学学报, 1988, 46, 301.); (j) Xiao, Z.; Hu, H.; Ma, J.; Chen, Q.; Guo, Y. Chin. J. Chem. 2013, 31, 939; (k) Su, Z.; Guo, Y.; Chen, Q.-Y.; Zhao, Z.-G.; Nian, B-Y. Chin. J. Chem. 2019, 37, 597.

[15] For selected examples of perfluoroalkylation of aromatic compounds with perfluoroalkyl iodides, see (a) Iqbal, N.; Choi, S.; Ko, E.; Cho, E. J. Tetrahedron Lett. 2012, 53, 2005; (b) Barata-Vallejo, S.; Flesia, M. M.; Lantaño, B.; Argüello, J. E.; Peñéñory, A. B.; Postigo, A. Eur. J. Org. Chem. 2013, 2013, 998; (c) Straathof, N. J. W.; Gemoets, H. P. L.; Wang, X.; Schouten, J. C.; Hessel, V.; Noël, T. ChemSusChem 2014, 7, 1612; (d) Huang, Y.; Lei, Y.-Y.; Zhao, L.; Gu, J.; Yao, Q.; Wang, Z.; Li, X.-F.; Zhang, X.; He, C.-Y. Chem. Commun. 2018, 54, 13662; (e) Yerien, D. E.; Cooke, M. V.; García Vior, M. C.; Barata- Vallejo, S.; Postigo, A. Org. Biomol. Chem. 2019, 17, 3741 .

[16] For selected examples of perfluoroalkylation of alkene with perfluoroalkyl iodides under visible light irradiation, see (a) Brace, N. O. J. Org. Chem. 1963, 28, 3093; (b) Habib, M. H.; Mallouk, T. E. J. Flurorine Chem. 1991, 53, 53; (c) Ogawa, A.; Imura, M.; Kamada, N.; Hirao, T. Tetrahedron Lett. 2001, 42, 2489; (d) Tsuchii, K.; Imura, M.; Kamada, N.; Hirao, T.; Ogawa, A. J. Org. Chem. 2004, 69, 6658; (e) Wallentin, C.-J.; Nguyen, J. D.; Finkbeiner, P.; Stephenson, C. R. J. J. Am. Chem. Soc. 2012, 134, 8875; (f) Mizuta, S.; Verhoog, S.; Engle, K. M.; Khotavivattana, T.; O’Duill, M.; Wheelhouse, K.; Rassias, G.; Médebielle, M.; Gouverneur, V. J. Am. Chem. Soc. 2013, 135, 2505; (g) Wang, Y.; Wang, J.; Li, G.-X.; He, G.; Chen, G. Org. Lett. 2017, 19, 1442; (h) Beniazza, R.; Remisse, L.; Jardel, D.; Lastécouères, D.; Vincent, J.-M. Chem. Commun. 2018, 54, 7451; (j) Rawner, T.; Lutsker, E.; Kaiser, C. A.; Reiser, O. ACS Catal. 2018, 8, 3950.

[17] For selected examples of transition metal catalyzed perfluoroalkylation of alkene with perfluoroalkyl iodides, see: (a) Gil-Rubio, J.; Guerrero-Leal, J.; Blaya, M.; Vicente, J.; Bautista, D.; Jones, P. G. Organometallics 2012, 31, 1287; (b) Blaya, M.; Bautista, D.; GilRubio, J.; Vicente, J. Organometallics 2017, 36, 1245; (c) Zheng, J.; Chen, P.; Yuan, Y.; Cheng, J. J. Org. Chem. 2017, 82, 5790.

[18] For selected reviews on the synthesis and application of 1 , 3-oxazines, see: (a) Schmidt, R. R. Synthesis 1972, 1972, 333; (b) Sato, M.; Sunami, S.; Kaneko, C. Heterocycles 1996, 42, 861.

[19] In the reaction of $O$-homoallyl benzimidates equipped with multisubstituted alkene and trichloroacetimidate analogue of $\mathbf{1}$, no desired product was detected, and most of the starting material remain unconsumed.

Unreactive substrates:
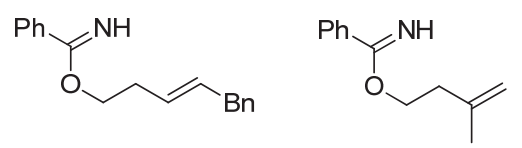